\title{
Rhesus Monkeys Use Geometric and Nongeometric Information During a Reorientation Task
}

\author{
S. Gouteux and C. Thinus-Blanc \\ Center for Research in Cognitive Neuroscience
}

\author{
J. Vauclair \\ Université de Provence
}

\begin{abstract}
Rhesus monkeys (Macaca mulata) were subjected to a place finding task in a rectangular room perfectly homogeneous and without distinctive featural information. Results of Experiment 1 show that monkeys rely on the large-scale geometry of the room to retrieve a food reward. Experiments 2 and 3 indicate that subjects use also nongeometric information (colored wall) to reorient. Data of Experiments 4 and 5 suggest that monkeys do not use small angular cues but that they are sensitive to the size of the cues (Experiments 6, 7, and 8). Our findings strengthen the idea that a mechanism based on the geometry of the environment is at work in several mammalian species. In addition, the present data offer new perspectives on spatial cognition in animals that are phylogenetically close to humans. Specifically, the joint use of both geometric and landmark-based cues by rhesus monkeys tends to demonstrate that spatial processing became more flexible with evolution.
\end{abstract}

Most of the studies of spatial representations in animals rely on the concept of cognitive map (Tolman, 1948), involving a kind of "bird's eye view" of the environment, which makes it possible to move efficiently in space between places charted on a map. Spatial behaviors of several species have been studied in such a perspective, with the aim to demonstrate that animals do have spatial representations endowed with adaptive properties. Indeed, many animal species appear to be able to construct and use cognitive maps to orient, ranging from fishes (Lopez, Broglio, Rodriguez, Thinus-Blacc, \& Salas, 1999) to monkeys (Joubert \& Vauclair, 1986; Menzel, 1973, 1978), though the most extensive studies have been conducted in rats at both the behavioral and brain level (see Thinus-Blacc, 1996, for review).

Many environmental features are likely to serve as constitutive elements of spatial representations, and understanding their nature is relevant for a more extensive analysis of the spatial mapping

S. Gouteux and C. Thinus-Blanc, Center for Research in Cognitive Neuroscience, National Center for Scientific Research, Marseille, France; J. Vauclair, Centre for Research in Cognition, Language, and Emotion, Université de Provence, Aix en Provence, France.

This research was supported by the Centre National de la Recherche Scientifique, by Grant 97-5-03224 from the Ministère de l'Education Nationale, de l'Enseignement Supérieur et de la Recherche and by Grant 1998 from the Société Francophone de Primatologie. We thank Bernard Arnaud for technical assistance and Alexa Riehle for allowing us to use her monkeys. In addition, we thank N. Martin and I.C. Paphaichiez for assistance with the experiments, V. Dasse for the statistical analysis, and Bruno Poucet and Simon Benhamou for useful comments on an earlier version of this article. Some of the experiments discussed in this article were previously presented at the sixth Annual Meeting of the Cognitive Neuroscience Society, April 1999, Washington, DC.

Correspondence concerning this article should be addressed to S. Gouteux, Center for Research in Cognitive Neuroscience, National Center for Scientific Research, 31 Chemin Joseph Aiguier, 13402 Marseille Cedex 20, France. Electronic mail may be sent to gouteux@lnf.cnrs-mrs.fr. process. Among these features, the geometry of surfaces defined by various types of discrete elements has recently been the target of an increasing number of studies (see below). However, some studies that belong to the traditional approach of cognitive maps have indirectly addressed this issue. For instance, in a series of reaction-to-change tests, hamsters explored four different objects in an open field. After habituation (quantified by the number and duration of contacts with the objects), a modification of the shape of the initial object configuration was brought about. Such changes induced a renewal of exploratory activity directed either selectively to the displaced objects or to all of them, even if the objects were identical (Thinus-Blacc et al., 1987). In contrast, modifying the size of the configuration had no effect. Such results demonstrate that hamsters had spontaneously encoded the geometry of the object arrangement, given that a modification of this feature induced strong reactions most likely being the result of the comparison between a stored representation of the initial arrangement and the perception of the new one. The lack of reexploration of the same configuration of a different size may correspond to the formation of a geometric category. Other authors have reached the same conclusion by using different kinds of situations (e.g., Greene \& Cook, 1997, in rats; Kamil \& Jones, 1997, in nutcrackers; Suzuki, Augerinos, \& Black, 1980).

Another means of tackling the problem of spatial processing in animals has been promoted by Cheng $(1986,1987)$ and Cheng and Gallistel (1984). These authors addressed the question of the encoding of two types of environmental features: geometric configuration and local cues. In a series of experiments, these authors have demonstrated that geometric features are spontaneously taken into account by rats and predominate over local cues, even when the latter would allow rats to make the distinction between two geometrically similar places, with only one being baited.

A distinctive feature of Cheng's experiments and of all the other ones discussed in this article, is that a disorientation procedure was conducted between the acquisition phase and the test, namely that 
the subject was rotated several times without vision. For instance, in Cheng's (1986) experiments, rats searched for food previously hidden in one of the four corners of a rectangular apparatus. After animals were familiarized with the experimental environment, they were removed from the apparatus, disoriented within a closed box, and returned to the apparatus to search for food. In this reorientation task, the rat had to reestablish its position and heading before it engaged in goal-directed behavior. To be oriented again, the rat could rely on the shape of the experimental apparatus, on the patterns, on the odors (if any), or on the brightness of the walls. Assuming that the oriented rat initially stored an internal representation (Tolman, 1948) of the food location (e.g., that the food was hidden near a particular cue or odor) and that the disoriented animal had to reorient itself to retrieve food (see also Gallistel, 1990; MacNaughton, Knierim, \& Wilson, 1994), Cheng reasonably concluded that the position where the rat searched for the reward indicates which spatial element the rat used to reorient itself.

In Cheng's (1986) experiments, rats showed a high rate of search both at the correct corner (the reward corner) and at the rotationally equivalent opposite corner, and these two corners on the same diagonal are defined by the same geometric relation within the experimental apparatus (length and width). This search pattern was constant during all experiments conducted by Cheng (1986, 1987), despite the availability of many cues (including obvious salient cues, such as strong distinctive odors, and large differences in contrast and luminosity) that could easily differentiate the two symmetrical locations. This finding suggests that rats were able to reorient in accord with the geometric relations provided by the environment. However, the rats seemed to be unable to take into account the nongeometric properties of that environment. Cheng concluded that the failure to reorient along nongeometric information stemmed from limits that are specific to the reorientation process. For Cheng (1986), reorientation in rats depended on a "geometric module," a task-specific, encapsulated system (see Fodor, 1983).

However, some of the results obtained by Cheng suggest that the greatest number of searches were conducted in the correct location, even when no significant difference was noted between the two geometrically correct corners. This tendency to retrieve the correct location could reflect a weak ability to use nongeometric information but could also result from an incomplete disorientation procedure of the rats. To distinguish between these two possibilities, Margules and Gallistel (1988) used a more rigorous disorientation procedure. Rats were first exposed to the reward location in an exposure box and then moved into another similar box (test box) that was differently located and oriented compared to the first one. This ingenious disorientation procedure led Margules and Gallistel to the same conclusion drawn by Cheng, namely that rats failed to use the nongeometric information to correctly locate the target. These findings, and similar findings from other investigators (Biegler \& Morris, 1993, 1996; Dudchenko, Goodridge, Seiterle, $\&$ Taube, 1997), provide further empirical data, showing that rats reoriented in accord with the shape of the environment but seemed to neglect nongeometric properties of the environment.

These findings stand in contrast with those gathered in the animal physiology literature, showing that rats can easily reorient and retrieve a goal by using different nongeometric information provided by the environment (see, e.g., Benhamou \& Poucet,
1998; Knierim, Kudrimoti, \& MacNaughton, 1995). Differences between the experimental environments, between procedures and motivational factors, could account for the discrepancy observed in the data (Hermer, 1997). For example, in studies by Cheng (1986), Hermer and Spelke (1994, 1996), or Biegler and Morris (1993, 1996), subjects were tested in apparatus with an informative shape (such as a rectangle). In contrast, other studies often placed rats in an environment with a minimal distinctive geometry (most often an enclosed cylinder). Another difference that may account for discrepant data is the level of familiarity with the test situation. Most often, in the studies about geometry, the subjects spend a relatively short time in test environments, whereas more classical studies have tested rats repeatedly in situations that become familiar through learning. It is thus likely that animals may use nongeometric information only when geometric information is minimized and when the cues have become stable and familiar. However, the use of geometric coding might have an adaptive significance for an animal.

Thus, Hermer and Spelke (1996) have provided an interesting ecoethological explanation for the predominant use of geometric information for reorientation in a natural environment. The macroscopic shape of the landscape does not change across seasons. In contrast, there are important changes in the nongeometric properties of the environment (e.g., the appearance of trees with or without leaves, or snowfall and melting). Thus, it is safer for animals to rely on geometric information that is not modified by seasonal changes. In a recent study (Gouteux, Vauclair, \& ThinusBlanc, 1999), using a reaction to spatial change procedure, we examined the spontaneous exploratory activity of four young baboons to determine the type of spatial coding (purely geometric or related to the identity of the objects) that was implemented while the animals were getting acquainted with an object configuration. During a habituation phase, all the subjects were individually familiarized with the initial spatial configuration (in an outdoor enclosure) made by the four objects of the configuration. We observed a decrease in the duration of contacts, indicating that the baboons got progressively familiarized with the initial situation. After habituation, animals were tested for their exploratory reactions (contact duration and order of spontaneous visits) to spatial changes brought about to the initial object configuration. Two kinds of spatial changes were made: a modification of (a) the shape of the configuration (by displacing one of the four different objects), and (b) the spatial arrangement without changing the initial shape (exchanging the location of two objects). In a second experiment, the four objects were identical, and a modification of the geometry was performed. Finally, in the third experiment, a substitution of a familiar object with a novel one was performed without changing the object configuration. Results showed that baboons strongly reacted to the geometric modifications of the configuration because they massively and selectively reexplored only the displaced object. In contrast, the baboons were less sensitive to changes of the local features that did not affect the initial spatial configuration. In this latter case, no specific spatial reexploration of the displaced objects was noticed. However, the results also suggest that the geometric encoding requires that the various elements that define the geometry of the explored space were not identical and that each of the various elements specifies a location to induce a selective reexploration directed toward the displaced objects. 
However, the inability to conjointly rely on geometry and nongeometric properties of the environment to reorient is not present in all vertebrates. Some avian species have been tested during a reorientation task adapted from Cheng's (1986) initial experiment. Results clearly demonstrated that chickens (Vallortigara, Zanforlin, \& Pasti, 1990) and pigeons (Kelly, Spetch, \& Heth, 1998), like rats and humans, use geometry to reorient. Surprisingly, these species were also able to use nongeometric spatial information to perfectly reorient. Hermer and Spelke $(1994,1996)$, in a series of studies conducted with human children, have examined the use of a geometric module by toddlers. Children (aged from 18 to 24 months) saw a desired toy that was being hidden in one of the comers of a rectangular homogeneous experimental chamber. After disorientation, the participants were asked to retrieve the toy. In one of the experiments, the chamber contained no distinctive landmark. In another one, a nongeometric feature (blue wall) that broke the symmetry of the experimental apparatus was added. Hermer and Spelke found that, when no information other than the shape of the environment was available, children searched equally often in the correct and in the rotationally equivalent corner, and more frequently in these two corners than in the other two remaining comers. When nongeometric information (a blue wall or a pair of toys placed in the room) was added, children still divided their searches between the two rotationally equivalent corners and seemed to ignore the added salient cues.

Hermer and Spelke concluded that young children do not use information other than the shape of the experimental environment to reorient, even when more salient nongeometric information was available and could help them to locate the correct corner. In the same study, Hermer and Spelke also tested human adults with the same experimental setup. Unlike children, adults were able to use both geometric and nongeometric information to optimize their search. Similar results have been found by Wang, Hermer, and Spelke (1999) in a square room; children (18-24 months old) used a distinctive geometric cue, but not a colored wall, to locate the hidden object, even though they had been familiarized with the colored wall over multiple training sessions.

These studies suggest that young children reorient by a process that is encapsulated, task-specific, and common to other mammals, whereas adults reorient in a more flexible way. To explain this source of flexibility, Spelke and Hermer (1996) argued that language is necessary to penetrate the geometric module and to allow for reorientation by integrating landmarks. More precisely, these authors proposed that the age at which children begin to successfully locate the target by using geometric and nongeometric information (at about 6-6.5 years of age; Hermer, unpublished data quoted by Spelke and Hermer, 1996) approximately corresponds to the age at which children begin to produce sentences that would uniquely specify object location and orientation (MacWhinney, 1991), such as "near the blue wall" or "to the right/left of the blue wall." Moreover, some interesting data show that when human adults perform the reorientation task previously mentioned, although they have to perform concurrent tasks (e.g., verbal shadowing), they are unable to use nongeometric information, but they still succeed in using geometric information (Hermer-Vasquez, Spelke, \& Katsnelson, 1999). According to Spelke and Hermer (1996), language seems to be necessary to bind the geometric module and other, nongeometric modules. Consequently, the use of both geometric information and local features in the environ- ment would constitute a specificity of adult humans' spatial cognitive abilities.

To our knowledge, no study has attempted to investigate this particular question in species other than rats (Cheng, 1986), birds (Vallortigara et al., 1990; Kelly et al., 1998), or human adults and children (Hermer \& Spelke, 1994, 1996). From a comparative point of view, it seems that the use of geometry to reorient is shared by several mammals. Along this perspective, we tested rhesus monkeys in the present experiments with the goal of examining whether nonhuman primates use a reorientation mechanism that is based on the geometry of the environment (Experiment 1) and whether such a mechanism is compatible with the use of nongeometric cues (Experiments 2 and 3). Because adult humans are able to linguistically encode the relevant information in the reorientation experiment, the relationship between spatial and object information can be encoded in other ways as well. Could a nonlinguistic animal solve tasks that require the integration of information from two distinct aspects of core knowledge? In another way, by testing nonhuman primates, these experiments allow us to test the language hypothesis, put forward by Hermer and Spelke, explaining why in a reorientation task, only human adults are able to conjointly use geometric and nongeometric spatial information. In addition, assuming that rhesus monkeys were able to use these two kinds of spatial information, we investigated to what extent monkeys could take into account local cues during their reorientation (Experiments 4 and 5) and also whether they were sensitive to the size and location of such cues in the experimental apparatus (Experiments 6, 7, and 8).

\section{Part 1}

We investigated the spatial reorientation abilities of nonhuman primates by using the geometric and nongeometric properties provided by a rectangular room. For that purpose, three experiments, adapted from the original experiment by Cheng (1986), were conducted with 3 rhesus monkeys. The first experiment was designed to test the reorientation of the monkeys when the only available spatial information was the shape of the apparatus. In the next two experiments, we investigated the use of geometric and nongeometric information, either associated to the rewarded box (Experiment 2) or dissociated from it (Experiment 3) in a task performed within the same rectangular room. The order of these experiments was counterbalanced across subjects.

\section{Experiment 1}

\section{Method}

Subjects. Three experimentally naive young rhesus monkeys (Macaca mulata) were individually studied. The group included 2 males (Orcas and Krill) and I female (Crevet). The subjects were $1.9(\mathrm{SD}=0.4)$ years of age at the beginning of the experiment. All monkeys were born in captivity at the Primatology Unit of the CNRS in Marseille, France, and they were housed in individual indoor cages during the experiments. They received food pellets and vegetables once a day in the afternoon.

Apparatus. Subjects were tested in a rectangular chamber $(4.0 \mathrm{~m}$ long X $2.5 \mathrm{~m}$ high X $2.0 \mathrm{~m}$ wide), filled with $3 \mathrm{~cm}$ of sawdust and perfectly homogeneous with respect to visual cues (see Figure 1). The apparatus was housed within a larger dark room with no obvious source of noise. It was composed of a metallic frame entirely recovered by white wooden panels. Two doors with an access hatch, opening at the center of each of the two 
longer walls, were present, but only one, because of technical problems, permitted entry into the apparatus. Four opaque identical boxes $(15 \mathrm{~cm}$ in diameter X $25 \mathrm{~cm}$ in height) stood in the four corners of the room. Each box could contain a food reward (Fruit Loops cereal), but the subjects could not locate the reward unless they looked into the box. The room was illuminated by a central overhead neon circular light $(100 \mathrm{~W})$. A videocamera, suspended from the center of the room's ceiling, provided an overhead view of the whole apparatus. At the center of the room, a transparent Plexiglas cage ( $40 \mathrm{~cm}$ wide X $45 \mathrm{~cm}$ long X $50 \mathrm{~cm}$ high) was fixed on a rotating support to allow the axial rotation of the cage. Above that central cage, curtains were added to prevent the monkey from seeing the experimental environment from inside the cage. The opening of the central cage was externally controlled by a mechanical system made of several pulleys and a cable that descended directly from the center of the room's ceiling. A white-noise generator was located on the ceiling of the apparatus, at the center, to prevent monkeys from maintaining their orientation through the use of acoustical external cues. Thus, the apparatus was symmetrical in its geometry.

Procedure. A training phase was conducted to familiarize the animals with the experimental apparatus and the experimental conditions. Each animal was daily captured and transported to the experimental room, using an opaque transport cage. Each monkey was transferred in the central transparent Plexiglas cage (darkened by black curtains) in such a way that the animal could never see the extra-apparatus environment. After a 1-min delay, the monkey was randomly released in front of one of the four walls of the room by the external mechanical system. The animal could then freely explore the experimental environment during a 10-min period and catch food rewards that had been previously laid out on the floor. After the 10-min delay, the access hatch was opened, and the monkey could return in the external opaque transport cage.

After 1 week of training (five sessions per day), the reward box was placed at one corner of the experimental room. That box was systematically rewarded, and the monkey was able to see the reward, which was being hidden in that box (curtains opened). The same procedure mentioned above was used: The monkey was released, and after a 10-min delay or immediately after the subject had retrieved the reward, it was returned to the outside opaque transport cage. The position of the corner reward box was randomly chosen for each monkey but was maintained constant during the experiment. Then, the three identical remaining boxes (not rewarded) were progressively added to the experimental room. In the same manner, the monkeys had to retrieve the reward previously indicated by the experimenter. The training phase ended when all monkeys systematically succeeded in retrieving the food reward. The delay to reach that level varied for each subject (from 2 days for Subject Krill to 2 weeks for Subject Crevet).

During Experiment 1, the monkeys were tested in the all-white condition (see Figure 2), and the same procedure was used for all of the experiments. As mentioned above, the subject was introduced to the central transparent Plexiglas cage with the curtains closed. After the opening of the curtains, the experimenter hid a reward in the same comer box that was rewarded during the training phase. For a given subject, the reward was hidden in the same location throughout the experiment to reduce extensive proactive interference. Then, the curtains of the central transparent Plexiglas cage were closed, and the monkey was disoriented by the experimenter (at least 10 full rotations of the cage), who was walking around the cage at varying speed to avoid serving as a landmark during disorientation. Then, the cage rotation was stopped, its entrance randomly facing one of the four walls of the rectangular chamber, and the experimenter continued walking around slowly so as not to cue the monkey to any possible location. Finally, the experimenter, without making any noise, left the experimental room through the access door. After a 10-s delay, the door of the central Plexiglas cage was opened from the outside, and the monkey could freely search for the reward box.

When the food was found, the access hatch was opened, and the monkey could return to the external opaque transport cage. Then, the subject was transferred again to the experimental room to be tested again. Ten trials a day during 5 consecutive days (50 trials) were conducted. A delay of $1 \mathrm{~min}$ occurred between each test trial.

Dependent measures. All search patterns were videotaped to later verify patterns of exploration made during the experiment. To count as a search, the monkey had to look inside the box or at least touch the box with its arm. A passage near the box or a look in its direction was not taken into

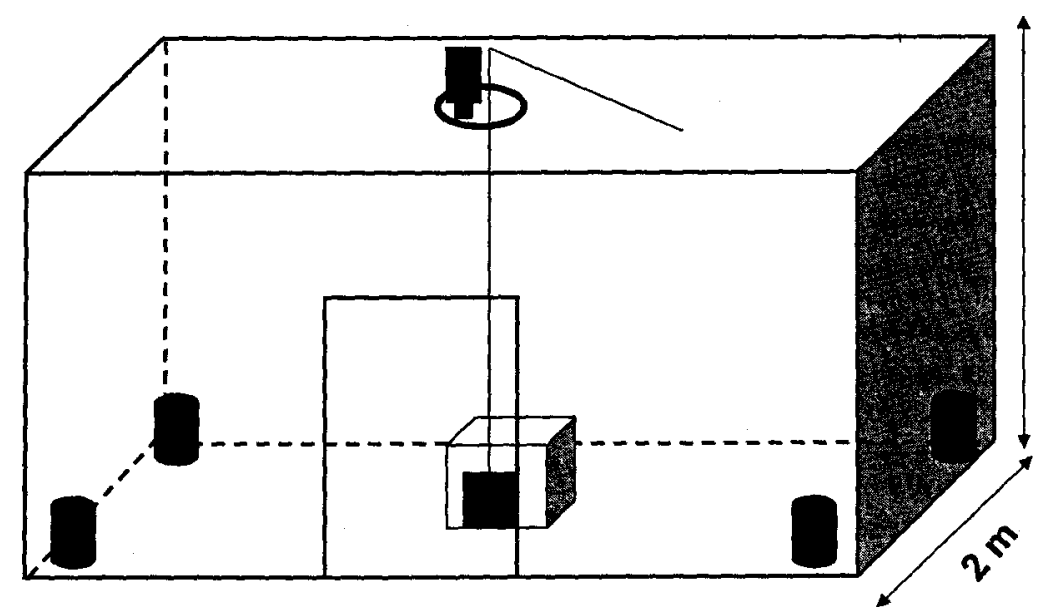

Figure 1. Schematic representation of the experimental environment ( $4.0 \mathrm{~m}$ long X $2.5 \mathrm{~m}$ high X $2.0 \mathrm{~m}$ wide). Subjects were introduced into the central Plexiglas cage, fixed on a rotative support (gray central box), and were released by an external mechanical command (full line drawn at the top of the ceiling), to retrieve the reward hidden in one of the four identical comer boxes (gray circular boxes). An overhead camera, a white-noise generator, and a circular light were affixed on the ceiling of the room (black rectangular box and black circle on the inn of the fiaurel 
1

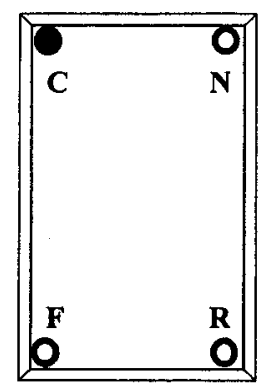

2

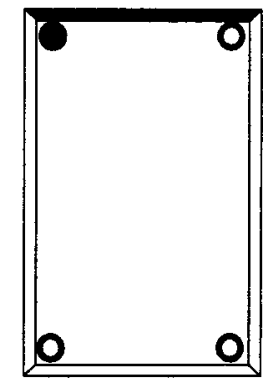

3

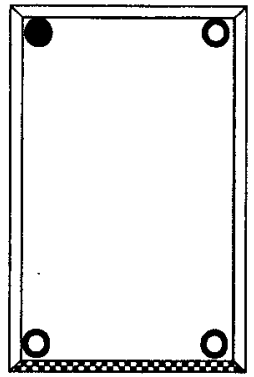

Figure 2. Overhead view of the testing environment for Experiment 1 (1, all-white condition), Experiment 2 (2, blue-wall condition), and Experiment 3 (3, checkered wall). The correct location (noted " $\mathrm{C}$ "), the rotational equivalent corner (noted "R"), and the geometrically inappropriate near (noted "N") and far (noted "F") corners are shown in the figure.

nd was unambiguous because the boxes were nearly as tall as was the ubject.

The principal analyses focused on the location of the monkey's first eacch choice during each of the 50 trials. Search location was coded in one of two categories (similar to those used by Hermer \& Spelke, 1994, 1996). $\mathrm{f}$ the subject searched in the correct box (rewarded box, noted "C" for 'correct") or if it was at the rotational equivalent box (at $180^{\circ}$ rotation hrough the center from the rewarded box, noted "R"), this response was onsidered as a geometrically appropriate choice. Other choices, near the eward box but geometrically inappropriate or far from the reward box but pometrically inappropriate (noted respectively, "N" and "F") were called ;eometrically inappropriate choices.

?esults

Table 1 presents the number of first choices performed by the 3 ubjects during the 50 trials. For each monkey, these data were ubjected to a chi-square one-sample test by which we compared he observed distribution to the theoretical frequency of an equal listribution in the "geometrically appropriate" and "geometrically nappropriate" above mentioned categories (i.e., $50 \%$ of chance for ach one). These results always reached statistical significance, )rcas, $\mathrm{X}(1, N=50)=20.49$; Krill, $\mathrm{X} 2(1, N=50)=46.80$; 'revet, $\mathrm{X} 2(1, N=50)=42.32 ; \mathrm{p}<.001$, indicating that the bserved distribution between these two categories was not deternined at random. A second chi-square one-sample test was com-

Cable 1

Vumber of Trials (Out of 50) During Experiment 1 for the subjects as a Function of Their Search Location (Correct, rotational, Geometrically Inappropriate Corners)

\begin{tabular}{lcccc}
\multicolumn{5}{c}{ Box } \\
\multicolumn{1}{c}{ Monkey } & $\mathrm{C}$ & $\mathrm{R}$ & $\mathrm{N}$ & $\mathrm{F}$ \\
)rcas & 21 & 20 & 4 & 5 \\
:rill & 25 & 24 & 1 & 0 \\
:revet & 23 & 25 & 2 & 0 \\
$\quad$ Average (\%) & 46 & 46 & 4.7 & 3.3
\end{tabular}

Vote. $\mathrm{C}=$ correct $; \mathrm{R}=$ rotational $; \mathrm{N}=$ near misses $; \mathrm{F}=$ far misses.
IJUIUU uu Liie UUM oouuucu III uic omerveu gevmemcauy appropriate category compared with an equal frequency of distributions of the searches to the two comers of that category (i.e., $50 \%$ of chance for each corner). This analysis indicates that the number of visits to Corner $\mathrm{C}$ was not statistically different from the number of visits to Corner R, Orcas, X2(1, $N=41)=0.02$; Krill, X2(1, $N$ $=49)=0.02 ;$ Crevet, $\mathrm{X} 2(1, N=48)=0.08 ; \mathrm{p}>.05$. This result shows that the correct and the geometrically equivalent corners were chosen with no specific preference.

Data analyses of the first 10 trials for each subject are reported in Table 2 (Experiment 1). These data were submitted to a logistic regression to test whether the scores for the 3 subjects, during the 10 first trials, show differences with the results of the entire experimental session (i.e., 50 trials). The analyses show that the performance of each subject is not statistically different within the same experiment, Orcas, $\mathrm{x}(1, N=10)=0.07$; Krill, $\mathrm{X} 2(1, N=$ $10)=0.01 ;$ Crevet, $\mathrm{X} 2(1, N=10)=0.25 ; \mathrm{p}>.05$. Thus, we can conclude that no improvement of the monkeys' performance takes place across trials.

\section{Discussion}

Results of Experiment 1 show that the monkeys rely on the geometry of the room to reorient. Indeed, in the present homogeneous environment (white-room condition), monkeys confined their search almost exclusively to both geometrically correct corners (the correct corner and the corner of the opposite side of the chamber), searching in these two locations with an equal frequency. Thus, like mature rats, toddlers, and adults, rhesus monkeys are able to reorient by reference to the shape of the environment (Cheng, 1986; Hermer \& Spelke, 1996).

Our results also indicate that the disorientation procedure used in this experiment is efficient and that no other landmark but the shape of the room is used by the subject to reorient in the experimental environment. Neither visual information within the experimental room nor nonvisual information from outside the room served as cues for reorientation. If any external or internal cue had been used, then monkeys would certainly have been able to locate the target without ambiguity. 
Table 2

Data Analysis of the 10 First Trials for Each Experiment and for the Subjects, Indicating Whether They Searched at the Correct Location, Made a Rotational Error, or Searched in the Geometrically Inappropriate Corners

\begin{tabular}{|c|c|c|c|}
\hline \multirow[b]{2}{*}{ Experiment and subject } & \multicolumn{3}{|c|}{ Box } \\
\hline & $\mathrm{C}$ & $\mathrm{R}$ & $\mathrm{N} \& \mathrm{~F}$ \\
\hline \multicolumn{4}{|l|}{ Experiment 1} \\
\hline Orcas & 4 & 5 & 1 \\
\hline Krill & 5 & 5 & 0 \\
\hline Crevet & 5 & 4 & 1 \\
\hline Average (\%) & 47 & 47 & 6 \\
\hline \multicolumn{4}{|l|}{ Experiment 2} \\
\hline Orcas & 7 & 2 & 1 \\
\hline Krill & 7 & 3 & 0 \\
\hline Crevet & 6 & 3 & 1 \\
\hline Average (\%) & 67 & 27 & 6 \\
\hline \multicolumn{4}{|l|}{ Experiment 3} \\
\hline Orcas & 7 & 1 & 2 \\
\hline Krill & 6 & 4 & 0 \\
\hline Crevet & 8 & 2 & 0 \\
\hline Average $(\%)$ & 70 & 24 & 6 \\
\hline \multicolumn{4}{|l|}{ Experiment 4} \\
\hline Orcas & 4 & 3 & 3 \\
\hline Krill & 6 & 4 & 0 \\
\hline Crevet & 6 & 4 & 0 \\
\hline Average (\%) & 53 & 37 & 10 \\
\hline \multicolumn{4}{|l|}{ Experiment 5} \\
\hline Orcas & 4 & 6 & 0 \\
\hline Krill & 5 & 5 & 0 \\
\hline Crevet & 4 & 6 & 0 \\
\hline Average (\%) & 43 & 57 & 0 \\
\hline \multicolumn{4}{|l|}{ Experiment 6} \\
\hline Krill & 5 & 3 & 2 \\
\hline Orcas & 4 & 5 & 1 \\
\hline Average $(\%)$ & 45 & 40 & 15 \\
\hline \multicolumn{4}{|l|}{ Experiment 7} \\
\hline Krill & 9 & 1 & 0 \\
\hline Crevet & 8 & 1 & 1 \\
\hline Average (\%) & 85 & 10 & 5 \\
\hline \multicolumn{4}{|l|}{ Experiment 8} \\
\hline Krill & 7 & 3 & 0 \\
\hline Crevet & 8 & 1 & 1 \\
\hline Average (\%) & 75 & 20 & 5 \\
\hline
\end{tabular}

Note. $C=$ correct $; \mathrm{R}=$ rotational $; \mathrm{N} \& \mathrm{~F}=$ near and far misses.

\section{Experiment 2}

In Experiment 2, we tested monkeys' ability to reorient along both the geometric shape and nongeometric properties of the experimental room, by using a local cue (colored wall) as in Hermer and Spelke's (1994) experiment.

\section{Method}

Subjects. The same subjects as in Experiment 1 were used. A delay of 1 day occurred between Experiment 1 and Experiment 2.

Apparatus. The apparatus was the same as in Experiment 1, but one of the small walls was completely covered by a blue panel (blue-wall condition). This landmark always occupied the same side with respect to the rewarded box (see Figure 2).

Procedure. The same procedure as in Experiment 1 was used, but a control phase was added. The control phase (10 trials at the end of the 50 experimental trials) consisted of making a $180^{\circ}$ transfer of the cue (blue wall) and of the rewarded box. The aim of that "rotation" was to check that the only information used by the monkeys to get reoriented was the blue wall in addition to the geometry of the experimental environment. Indeed, if during the 50 experimental trials, an uncontrolled cue (from inside or outside the apparatus) served as a landmark and guided the subject to the reward box, then the rotation of the landmark would have eliminated the use of such a cue.

\section{Results}

Table 3 presents the number of first searches performed by the 3 subjects during the 50 test trials. For each monkey, the data of the test were subjected to a chi-square one-sample test by which we compared the observed distribution to the theoretical frequency of an equal distribution in the geometrically appropriate and geometrically inappropriate above-mentioned categories (i.e., $50 \%$ of chance for each one). These results always reached a statistically significant level, Orcas, $\mathrm{X} 2(1, N=50)=32.0$; Krill, $\mathrm{X} 2(1, N=50)$ $=46.1$; Crevet, $X 2(1, N=50)=46.1$; all ps $<.001$. Another chi-square one-sample test computed on the data obtained in the two corners of the observed geometrically appropriate category compared with an equal frequency of distributions of the searches to these two corners (i.e., $50 \%$ of chance for each one), indicated that the numbers of visits to Corner $\mathrm{C}$ and to Corner $\mathrm{R}$ were statistically different, Orcas, A, $2(1, N=45)=18.68, \mathrm{p}<.001$; Krill, X2 $(1, N=49)=9.00, \mathrm{p}<.005$; Crevet, $\mathrm{X} 2(1, N=49)=$ $22.22 ; \mathrm{p}<.001$. The monkeys mainly chose the correct corner.

For Experiment 2, the data of each subject during the entire experimental session (50 trials) are very similar to those observed during the first 10 trials (see Table 2, Experiment 2). Thus, we can conclude that no obvious improvement of the monkeys' performance takes place during the experimental procedure, logistic regression: Orcas, $\mathrm{X}(1, N=60)=0.05 ; \mathrm{Krill}, \mathrm{X} 2(\mathrm{l}, N=60)$ 0.01 ; Crevet, $\mathrm{X} 2(1, N=60)=4.70 ; \mathrm{p}>.05$.

During the virtual rotation control session, all the subjects reacted in the same way. On average, $84 \%$ of their first choices during the 10 trials were directed to the rewarded box (noted "C"; 90\% for Subject Orcas, $60 \%$ for Subject Krill, and 99\% for Subject Crevet). The geometrically equivalent corner (Corner $\mathrm{R})$ received an average of $13 \%$ of the first visits $(0 \%$ for Orcas, $30 \%$ for Krill, and $1 \%$ for Crevet). Finally, the geometrically inappropriate corners (noted "N" and " $\mathrm{F}$, ,) received an average of $3 \%(0 \%$ for Orcas, $10 \%$ for Krill, and $0 \%$ for Crevet) of the first visits. This

Table 3

Number of Trials (Out of 50) During Experiment 2 for the Subjects as a Function of Their Search Location (Correct, Rotational, Geometrically Inappropriate Corners)

$$
\text { Box }
$$

\begin{tabular}{llrll}
\multicolumn{1}{c}{ Monkey } & $\mathrm{C}$ & $\mathrm{R}$ & $\mathrm{N}$ & $\mathrm{F}$ \\
\hline Orcas & 37 & 8 & 4 & 1 \\
Krill & 35 & 14 & $\mathrm{I}$ & 0 \\
Crevet & 41 & 8 & 0 & 1 \\
$\quad$ Average (\%) & 75 & 20 & 3.5 & 1.5
\end{tabular}

Note. $\quad C=$ correct $; \mathrm{R}=$ rotational $; \mathrm{N}=$ near misses $; \mathrm{F}=$ far misses. 


\section{SPATIAL REORIENTATION IN MONKEYS}

result suggests that the subjects used no cue (inside or outside the apparatus) other than the blue wall to reorient.

\section{Discussion}

The present results are in agreement with those obtained with human adult subjects (Hermer \& Spelke, 1994, 1996). They clearly indicate that monkeys confined their search to the correct corner box with high consistency. Because only the presence of the blue wall distinguished this environment from the homogeneous environment of Experiment 1, and because the control session was successfully realized, this finding indicates that our monkeys were still able to take into account the nongeometric property of the environment in their search strategy.

However, monkeys may have located the rewarded box either by a combined use of geometric and blue-wall information (by simultaneously noticing that the reward was hidden in a corner with appropriate geometry and that it was hidden near the blue wall) or by directly encoding the relation of the reward to the blue wall (by noticing that the object was hidden to the left or right of the blue wall). To test for these two possibilities, we dissociated, in a third experiment, the reward box and the landmark to see whether the monkeys were able to retrieve the correct box.

\section{Experiment 3}

In Experiment 3, we tested the monkeys' ability to reorient in accord with both the shape and the nongeometric cue of the room when this cue was not directly associated with the reward.

\section{Method}

Subjects. The same 3 subjects were used in Experiment 3. Between Experiments 2 and 3, a delay of 1 month elapsed.

Apparatus. The apparatus was the same as used in Experiment 2 except that one of the small walls of the apparatus, instead of a plain blue panel, was checkered by several black panels $(65 \mathrm{~cm} \times 60 \mathrm{~cm})$. The panels always occupied the opposite side with respect to the rewarded box (see Figure 2).

Procedure. The same procedure as in Experiment 2 was used, including the 10 control trials at the end of the experiment.

\section{Results}

Table 4 presents the number of first searches performed by the 3 subjects during the 50 test trials of Experiment 3. For each mon-

Table 4

Number of Trials (Out of SO) During Experiment 3 for the Subjects as a Function of Their Search Location (Correct, Rotational, Geometrically Inappropriate Corners)

Box

\begin{tabular}{llrll}
\multicolumn{1}{c}{ Monkey } & $\mathrm{C}$ & $\mathrm{R}$ & $\mathrm{N}$ & $\mathrm{F}$ \\
\hline Orcas & 41 & 6 & 3 & 1 \\
Krill & 34 & 16 & 0 & 0 \\
Crever & 45 & 4 & 0 & 1 \\
$\quad$ Average (\%) & 80 & 17 & 2 & 1
\end{tabular}

Note. $C=$ correct $; \mathrm{R}=$ rotational; $\mathrm{N}=$ near misses; $\mathrm{F}=$ far misses. key, the data were subjected to a chi-square one-sample test in which we compared the observed distribution to the theoretical frequency of an equal distribution in the geometrically appropriate and geometrically inappropriate categories (i.e., $50 \%$ of chance for each one). These test results always reached a statistically significant level, Orcas, $\mathrm{X} 2(1, \mathrm{~N}=50)=38.7$; Krill, $\mathrm{X} 2(l, N=50)=$ 50.0; Crevet, $X 2(1, N=50)=46.1$, all ps $<.001$. A second chi-square one-sample test computed on the data obtained in the observed geometrically appropriate category compared with an equal frequency of distribution of the two corners of that category (i.e., $50 \%$ of chance for each one) indicated that the number of visits to Comer $\mathrm{C}$ and to Corner $\mathrm{R}$ were statistically different, Orcas, $X(1, N=47)=26.06, \mathrm{p}<.001 ;$ Krill, $\mathrm{X} 2(1, N=50)=$ 6.48, p <.025; Crevet, $X 2(l, N=49)=34.30, \mathrm{p}<.001$.

For Experiment 3, the results obtained by each subject, during the entire experimental session (50 trials), are not statistically different to those observed during the first 10 trials (see Table 2, Experiment 3), Orcas, X2 $(1, N=60)=1.67$; Krill, X2 $(1, N=60)$ $=0.36$; Crevet, $X 2(l, N=60)=0.48 ; \mathrm{p}>.05$. Thus, we can conclude that no obvious improvement of the monkeys' performance takes place across trials.

During the virtual rotation control session, all the subjects reacted in the same way. On average, $83 \%$ of their first choices during the 10 sessions were directed to the rewarded box (box noted "C"; $90 \%$ for Orcas, $80 \%$ for Krill, and $80 \%$ for Crevet). The geometrically equivalent comer (Corner $\mathrm{R}$ ) received $13 \%$ of the first visits on average ( $0 \%$ for Orcas, $20 \%$ for Krill, and $20 \%$ for Crevet). Finally, the geometrically inappropriate corners (noted "N" and "F") received an average of 3\% of the first visits (10\% for Subject Orcas, 0\% for Subject Krill, and 0\% for Subject Crevet).

\section{Discussion}

As in the previous experiment, the correct corner box was predominantly chosen, in preference to the rotational equivalent corner. Our data confirm that monkeys are able, like human adults, to combine both the geometric properties of the room and nongeometric information to locate a target when they are disoriented. Moreover, in this experiment, nongeometric information was not directly associated with the rewarded box. Thus, we can conclude that monkeys located the rewarded box by combining both geometric and nongeometric information instead of directly encoding the relation of the reward to the landmark (i.e., by considering that the reward was hidden to the left or right of the landmark).

\section{Part 2}

Assuming that the monkeys were able to use both geometric and nongeometric information to reorient themselves in the experimental room (as shown by the previous experiments), we further investigated that specific spatial ability. Thus, Experiments 4 and 5 were designed to determine whether the monkeys are sensitive to more local spatial information, such as corner cues, and whether they are also able to use local cues dissociated from the goal (distal cues) to orient themselves.

\section{Experiment 4}

Experiment 4 was aimed at determining whether monkeys use four distinctive small corner panels to specify a place, and, 
whether the location of featural information (near or far from the target location) is crucial for disambiguating geometrically equivalent comers. For that purpose, the four landmark panels were located at each comer, during the presentation phase (before disorientation). For the test trials and before the disorientation, the panels at the correct corner and at its diagonally opposite corner were removed. Consequently, there was no distinctive featurai information to the correct corner and to its diagonal opposite. Only landmarks located at the other two corners could be used to differentiate the baited comer from its diagonal opposite, as it was the case for Cheng's (1986) experiments.

\section{Method}

Subjects. The same 3 subjects were used in this experiment. A delay of 1 month occurred between Experiments 3 and 4 .

Apparatus. The apparatus was the same as used in Experiment 1 (all-white condition) but a specific landmark was located at each corner of the experimental room. Landmarks had a similar shape and size (32 $\mathrm{cm} \times 23 \mathrm{~cm}$ ) but different colors (red, yellow, blue, and green) and different patterns (horizontal lines, crosses, circles, and squares) were used (see Figure 3).

Procedure. The same procedure as in the previous experiments was used, except that during the disorientation procedure, the two landmarks of the geometrically correct corners were removed.

\section{Results}

Table 5 presents the number of first-choice searches performed by the 3 subjects during the 50 test trials. For each monkey, the data for the test were subjected to a chi-square one-sample test by which we compared the observed distribution in the geometrically appropriate and geometrically inappropriate categories to the theoretical frequency of an equal distribution of these two categories (i.e., $50 \%$ of chance for each one). These results always reached statistical significance, Orcas, $X(1, N=50)=23.1$; Krill, X2(1, N $=50)=15.7$; Crevet, $X 2(1, N=50)=50.0 ; \mathrm{p}<.001$. A second chi-square one-sample test computed on the data obtained in the observed geometrically appropriate category compared with an equal frequency of distribution of the two comers' choices of that category (i.e., $50 \%$ of chance for Comers $\mathrm{C}$ and $\mathrm{R}$ ) indicated that the number of visits to the geometrically appropriate comers were not statistically different, Orcas, $\mathrm{X}(1, N=42)=2.38$; Krill, X2(1, $N=39)=1.24$; Crevet, $\mathrm{X} 2(1, N=50)=0.32 ; \mathrm{p}>.05$

Data analysis of the 10 first trials for each subject are reported in Table 2 (Experiment 4). The score for the 3 subjects for the 10 first trials shows no statistical differences with the results of the entire experimental session (i.e., 50 trials), Orcas, $X(1, N=10)$ $=1.82 ;$ Krill, $\mathrm{X} 2(1, N=10)=2.45 ;$ Crevet, $\mathrm{X} 2(1, N=10)=0.18$; $\mathrm{p}>.05$. Thus, we can conclude that no obvious improvement of the monkeys' performance takes place during the experimental procedure.

\section{Discussion}

The results of Experiment 4 indicate that monkeys were not able to use small-size distal information to retrieve the rewarded box. However, they still relied on the geometry of the apparatus because they searched as often in the correct box as in the geometrically equivalent one. An alternative explanation is that both the small size of the landmarks and their location, dissociated from the baited comer, may have rendered the task too difficult. Thus, to test for this hypothesis, we ran another experiment (Experiment 5) where four different panels remained during the test phase (after disorientation).

\section{Experiment 5}

Because the monkeys failed to retrieve the reward box by using the featural information distant from the geometrically correct location, in Experiment 5, we tested whether monkeys were able to use four small different landmark panels to reorient and find the reward. In that experiment, each corner was individualized by a featural panel, during the whole experiment. If monkeys used the local cues to reorient, then they should have increased their performance by finding the correct box during their first search.

\section{Method}

Subjects. The same 3 subjects were used in this experiment. A delay of 1 month occurred between Experiments 4 and 5 .
4

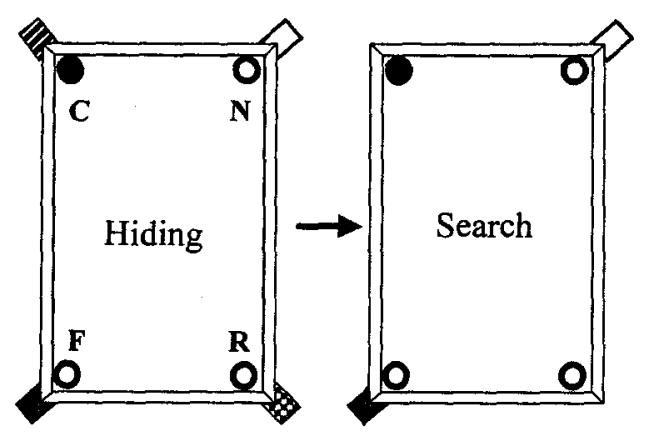

5

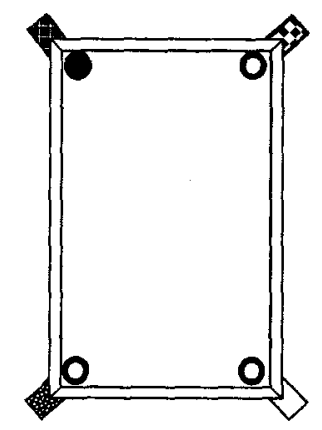

Figure 3. Overhead view of the testing environment for Experiments 4 and 5. Each corner is identified by a unique nongeometric colored pattern. In Experiment 4, the two patterns directly associated to the rewarded corner and to the geometrically equivalent comer were removed during subjects' disorientation. The correct location (noted "C"), the rotational equivalent corner (noted "R"), and the geometrically inappropriate near (noted " $N$ ") and far (noted " $F$ ") comers are shown in the figure. 
Table 5

Number of Trials (Out of 50) During Experiment 4 for the Subjects as a Function of Their Search Location (Correct, Rotational, Geometrically Inappropriate Corners)

\begin{tabular}{|c|c|c|c|c|}
\hline \multirow[b]{2}{*}{ Monkey } & \multicolumn{4}{|c|}{ Box } \\
\hline & $\mathrm{C}$ & $\mathrm{R}$ & $\mathrm{N}$ & $\mathrm{F}$ \\
\hline Orcas & 26 & 16 & 3 & 5 \\
\hline Krill & 23 & 16 & 6 & 5 \\
\hline Crever & 27 & 23 & 0 & 0 \\
\hline Average (\%) & 51 & 37 & 6 & 6 \\
\hline
\end{tabular}

Note. $C=$ correct $; \mathrm{R}=$ rotational $; \mathrm{N}=$ near misses $; \mathrm{F}=$ far misses.

Apparatus. The apparatus was the same as used in Experiment 1 (all-white condition). A landmark was fixed in each comer of the experimental room. Each landmark had a similar shape and size $(32 \mathrm{~cm} \mathrm{X} 32 \mathrm{~cm})$ but had different colors (red, yellow, blue, and green) and different patterns (horizontal lines, crosses, circles, and squares). Thus, each comer had a specific identifying cue (see Figure 3), different from those used in the previous experiment.

Procedure. The same procedure was used as in Experiment 2.

\section{Results}

Table 6 presents the number of first-choice searches performed by the 3 subjects during the 50 test trials. For each monkey, test data were subjected to a chi-square one-sample test by which we compared the observed distribution in the geometrically appropriate and geometrically inappropriate categories to the theoretical frequency of an equal distribution of these two categories (i.e., $50 \%$ of chance for each one). These results always reached statistical significance, Orcas, $\mathrm{X}(1, N=50)=32.0 ;$ Krill, $\mathrm{X} 2(1, N=$ $50)=35.3$; Crevet, $\mathrm{X}(1, N=50)=50.0 ; \mathrm{p}<.001$. A second chi-square one-sample test computed on the data obtained in the observed geometrically appropriate category (Comers C and R) compared with an equal frequency of distributions of searches to the two corners (i.e., $50 \%$ of chance for each one) indicated that the number of visits to Comer $\mathrm{C}$ and to Corner $\mathrm{R}$ were not statistically different, Orcas, X2(1, N=45) =0.55; Krill, X2(1, $N$ $=47)=2.57$; Crevet, $12(1, N=50)=0.01 ; \mathrm{p}>.05$.

Concerning the data of the first 10 trials (see Table 2, Experiment 5), we notice that the individual results obtained during these first trials are not statistically different from the results observed during the 50 trials of this experiment, Orcas,, $1_{2}(1, N=60)=$ 0.31 ; Krill, X2 $(1, N=60)=0.01$; Crevet, X2(1, $N=60)=0.32$; $\mathrm{p}>.05$. Thus, the subjects have not improved their performance over the trials of Experiment 5.

\section{Discussion}

As in the previous experiment, monkeys were not able to correctly locate the target. Obviously, they still relied only on the geometric properties of the apparatus for locating the rewarded box. Thus, from the two above experiments, we can conclude that small local landmarks are not used by monkeys to orient themselves in our environment. Even if the cues we used were salient and very distinctive, the monkeys did not use them during their orientation. It seems that cues need to have specific properties to be meaningful. We can also wonder whether the location of the cue is important to guide the monkey after being disoriented. In Experiments 2 and 3, the cue was located on one of the small walls of the apparatus (width), and, even if it was occupying the main part of that wall, we cannot be certain about what characteristics the monkeys used to orient (the location of the cue relative to the wall or its proper physical features).

\section{Part 3}

Because the monkeys were not able to reorient with the corner landmark in the previous experiment and because in Experiments 2 and 3 they were able to use a nongeometric feature, the next experiments further investigated the influence of the size and location of landmarks used by monkeys to reorient. Using the same orientation task in Experiment 6, we tested whether monkeys could rely on a cue smaller than that used in Experiments 2 and 3. The same question was addressed in Experiment 7 but with a larger cue, and also in Experiment 8, with different cues' location. The order of these experiments was counterbalanced across subjects for Experiments 6 and 7.

\section{Experiment 6}

In this experiment, we tested the monkeys' abilities to reorient in the rectangular environment, relying on both the shape of the room and a small nongeometric cue to determine the importance of the size of the landmark for the subjects.

\section{Method}

Subjects. Because 1 subject became bored and stopped searching, only 2 out of the 3 animals (Orcas and Krill) were able to complete this experiment. A delay of 1 month occurred between Experiments 5 and 6.

Apparatus. The apparatus was the same as in Experiment 2, but the landmark used in this experiment was a small colored panel $(21 \mathrm{~cm} \times 30$ $\mathrm{cm})$ with different patterns. Each monkey had its own cue with one color and one pattern, randomly chosen. The cue was located in the middle of the small side of the experimental apparatus (the one with the rewarded box). To be sure that the subject would see it, we placed it at the same height as the boxes (see Figure 4).

Procedure. The same procedure as in Experiment 5 was used.

\section{Results}

Table 7 presents the number of first-choice searches performed by the 2 subjects during the 50 test trials. For each monkey, the

Table 6

Number of Trials (Out of 50) During Experiment $S$ for the Subjects as a Function of Their Search Location (Correct, Rotational, Geometrically Inappropriate Corners)

Box

\begin{tabular}{lllll}
\multicolumn{1}{c}{ Monkey } & $\mathrm{C}$ & $\mathrm{R}$ & $\mathrm{N}$ & $\mathrm{F}$ \\
\hline Orcas & 20 & 25 & 3 & 2 \\
Krill & 29 & 18 & 1 & 2 \\
Crevet & 26 & 24 & 0 & 0 \\
$\quad$ Average (\%) & 50 & 45 & 2.5 & 2.5
\end{tabular}

Note. $\quad C=$ correct $; \mathrm{R}=$ rotational $; \mathrm{N}=$ near misses; $\mathrm{F}=$ far misses. 

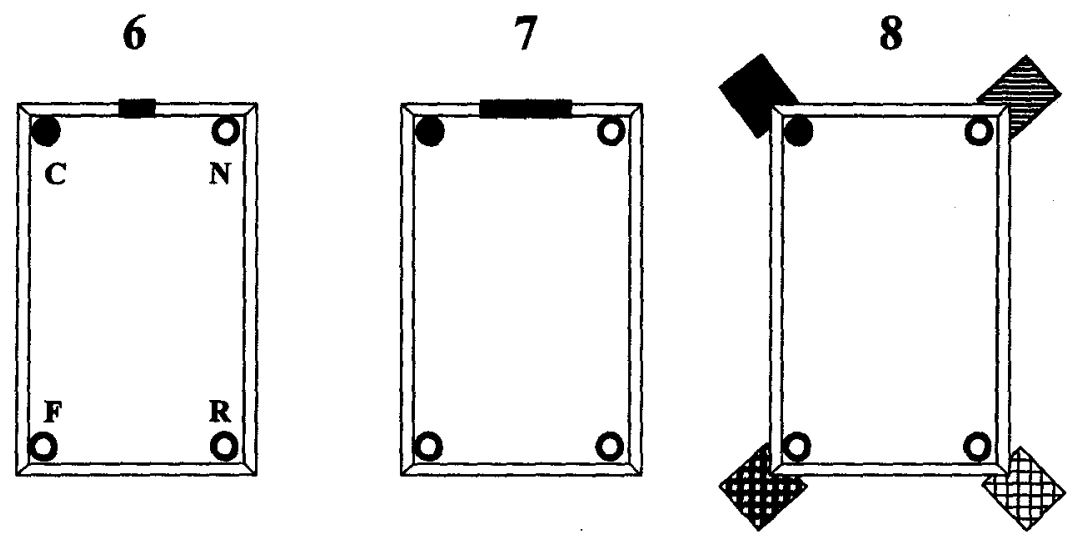

\begin{abstract}
Figure 4. Overhead view of the testing environment for Experiments 6 (small-size cue), 7 (intermediate-size cue), and 8 . The correct location (noted " $C$ "), the rotational equivalent corner (noted "R"), and the geometrically inappropriate near (noted " $N$ ") and far (noted " $F$ ") corners are shown in the figure.
\end{abstract}

data were subjected to a chi-square one-sample test in which we compared the observed distribution in the geometrically appropriate and geometrically inappropriate categories to the theoretical frequency of an equal distribution of these two categories (i.e., $50 \%$ of chance for each one). These results always reached statistical significance, Orcas, $\mathrm{X} 2(1, N=50)=6.5, \mathrm{p}<.001$; Krill, $\mathrm{X} 2(1, N=50)=20.48, \mathrm{p}<.005$. A second chi-square one-sample test computed on the data obtained in the observed geometrically appropriate category (Comers $\mathrm{C}$ and $\mathrm{R}$ ), compared with an equal frequency of distribution of the searches to these two corners (i.e., $50^{\circ} 10$ of chance for each one), indicated that the number of visits to Corner $\mathrm{C}$ and to Corner $\mathrm{R}$ were not statistically different, Orcas, , $2(1, N=34)=1.06$; Krill, $X 2(l, N=41)=0.22 ; \mathrm{p}>.05$.

Table 2 (Experiment 6 ) indicates the results for each subject during the 10 fast trials. The individual scores obtained for these 10 first trials do not statistically differ from the scores performed during the 50 trials of Experiment 6, Orcas, $X_{2}=0.78$; Krill, $\mathrm{X} 2=0.01 ; \mathrm{p}>.05$. These results indicate that no improvement of the monkeys' performance occurred during Experiment 6.

\section{Discussion}

The monkeys were not able to disambiguate the correct comer from the geometrical one by using the single small cue. They still relied exclusively on the geometric properties of the environment to reorient. Because the cue was of the same size as the corner cues

Table 7

Number of Trials (Out of 50) During Experiment 6 for the Subjects as a Function of Their Search Location (Correct, Rotational, Geometrically Inappropriate Corners)

\begin{tabular}{lccrr} 
& \multicolumn{5}{c}{ Box } \\
\multicolumn{1}{c}{ Monkey } & C & $R$ & $N$ & F \\
\hline Orcas & 20 & 14 & 8 & 8 \\
Krill & 22 & 19 & 6 & 3 \\
$\quad$ Average (\%) & 42 & 33 & 14 & 11
\end{tabular}

Note. $C=$ correct $R=$ rotational $; \mathrm{N}=$ near misses; $\mathrm{F}=$ far misses.
(Experiment 5), but located on one of the small sides of the room (the side where the blue wall had been affixed in Experiment 2), it thus appears that the size but not the location of the cue was useful for monkeys to reorient.

\section{Experiment 7}

The purpose of this experiment was to further examine the conclusion of Experiment 6, that is, whether the size of the cue is important for the monkeys. Thus, we tested the monkeys by using an intermediate landmark size, between those used in Experiments 2 and 3 (large) and in Experiment 6 (small).

\section{Method}

Subjects. Experiment 7 was run with 2 subjects because Subject Orcas stopped searching for reward. Between Experiments 6 and 7, a delay of 1 week occurred.

Apparatus. The apparatus was the same as used in Experiment 6 except that the landmark used in that experiment was of intermediate size $(50 \mathrm{~cm} \times 60 \mathrm{~cm})$ between the large one used in Experiment 2 and the small one used in Experiment 6 (see Figure 4).

Procedure. The procedure was identical to the one used in the previous experiment except that a 10-trial control session was given at the end of the 50 trials run per each subject. It consisted of the same $180^{\circ}$ rotation of the landmark and the rewarded box as in Experiment 2. The purpose of this control session was to check that the information used by the subjects to reorient was only the colored cue, together with the geometry of the experimental apparatus.

\section{Results}

Table 8 presents the number of first-choice searches performed by the 2 subjects during the 50 test trials. For each monkey, the data were subjected to a chi-square one-sample test in which we compared the observed distribution in the geometrically appropriate and geometrically inappropriate categories to the theoretical frequency of an equal distribution of these two categories (i.e., $50 \%$ of chance for each one). These results always reached a statistically significant level, Crevet, X2 $(1, N=50)=36.28$; Krill, $\mathrm{Al}, N=50)=42.32 ; \mathrm{p}<.001$. A second chi-square one-sample 
Table 8

Number of Trials (Out of 50) During Experiment 7 for the Subjects as a Function of Their Search Location (Correct, Rotational, Geometrically Inappropriate Corners)

\begin{tabular}{llrrr}
\multicolumn{5}{c}{ Box } \\
\multicolumn{1}{c}{ Monkey } & $\mathrm{C}$ & $\mathrm{R}$ & $\mathrm{N}$ & $\mathrm{F}$ \\
\hline Krill & 36 & 12 & 1 & 1 \\
Crever & 40 & 6 & 2 & 2 \\
$\quad$ Average (\%) & 76 & 18 & 3 & 3
\end{tabular}

Note. $\mathrm{C}=$ correct $; \mathrm{R}=$ rotational $; \mathrm{N}=$ near misses $; \mathrm{F}=$ far misses.

test computed on the data obtained in the observed geometrically appropriate category (Comers $\mathrm{C}$ and $\mathrm{R}$ ), compared with an equal frequency of distribution of the searches to these two corners (i.e., $50 \%$ of chance for each one), indicated that the number of visits to Comer $\mathrm{C}$ and to Comer R were statistically different, Crevet, X2(1, $N=48)=25.13 ;$ Krill, $\sim 2(1, N=46)=12.00 ; \mathrm{p}<.001$, showing that the monkey correctly located the reward after being disoriented.

The subject reacted in the same way during the virtual rotation control session. On average, $80 \%$ of the first visits during the 10 trials were focused on the rewarded box (Box C: $70 \%$ for Krill and $90 \%$ for Orcas). The geometrically equivalent comer (Comer R) received on average $15 \%$ of the first visits ( $20 \%$ for Krill and $10 \%$ for Orcas). Finally, the geometrically inappropriate comers (noted "N' and "F,,) received on average $5 \%$ of the first visits ( $10 \%$ for Krill and $0 \%$ for Orcas).

As for the previous experiments, the results of the first 10 trials for each subject are shown in Table 2 (Experiment 7). Analysis of the individual data compared with the performance obtained for the 50 trials of Experiment 7 indicates no statistical difference, Krill, $\mathrm{X}(1, N=60)=2.58$; Crevet, $\mathrm{X} 2(1, N=60)=0.01 ; p>$ .05 . These results show no evidence for an improvement of the performance across the experimental session.

\section{Discussion}

The monkeys were able to search in the correct corner as early as the fast trial. Thus, they must have used the single cue to reorient and to disambiguate the correct corner from the geometrically opposite corner. The control condition confirms that no other cue than the landmark, inside or outside of the apparatus, was used by the subjects to retrieve the target.

\section{Experiment 8}

This last experiment was run to test the importance of the cue size compared with its location (comer or wall) for reorientation. For that purpose, we decided to test the subjects with the same angular cue configuration used in Experiment 5 but with a larger cue size. If the cue size is important (and not its location), then the subjects are expected to succeed in correctly locating the rewarded corner in contrast with what subjects did in Experiment 5.

\section{Method}

Subjects. As in the previous experiment, only 2 subjects passed this experiment because Subject Orcas stopped searching for the reward. Between Experiments 7 and 8, a delay of 2 months occurred.
Apparatus. The same apparatus as in Experiment 5 was employed, except that the landmark used in that experiment was of intermediate size $(50 \mathrm{~cm} \mathrm{X} 60 \mathrm{~cm})$ between the large one used in Experiment 2 and the small one used in Experiment 6 (see Figure 4). The cues were located in each one of the four comers of the experimental apparatus.

Procedure. The procedure was identical to the one used in the previous experiments, except that a 10 -trial control session was given at the end of the 50 trials run per each subject. It consisted of a $180^{\circ}$ rotation of the four corner cues and the rewarded box as in Experiment 2. The purpose of this control session was to check that the information used by the subjects to reorient was only provided by geometry of the room and the four different corner cues.

\section{Results}

Table 9 presents the number of first-choice searches performed by the 2 subjects during the 50 test trials. For each monkey, the data were subjected to a chi-square one-sample test in which we compared the observed distribution in the geometrically appropriate and geometrically inappropriate categories to the theoretical frequency of an equal distribution of these two categories (i.e., $50 \%$ of chance for each one). These results always reached statistical significance, Crevet, $\mathrm{X} 2(\mathrm{l}, N=50)=32.00, \mathrm{p}<.001$; Krill, $\mathrm{X} 2(1, N=50)=28.88, \mathrm{p}<.001$. A second chi-square one-sample test computed on the data obtained in the observed geometrically appropriate category (Corners $\mathrm{C}$ and $\mathrm{R}$ ), compared with an equal frequency of distribution of the searches to these two corners (i.e., $50 \%$ of chance for each one), indicated that the number of visits to Corner $\mathrm{C}$ and to Corner $\mathrm{R}$ were statistically different, Crevet, X2(1, $N=45)=21.35, \mathrm{p}<.001 ;$ Krill, $, \mathrm{y} 2(1, N=44)=13.1, \mathrm{p}<.001$. This suggests that monkeys mainly chose the correct comer.

During the virtual rotation control session, all subjects reacted in the same way. On average, $85 \%$ of their first choice during the 10 control trials were directed to the rewarded box (noted "C"; $80 \%$ for Subject Crevet and 90\% for Subject Krill). The geometrically equivalent comer (noted "R") received an average of $10 \%$ of the first visits (20\% for Subject Crevet and $0 \%$ for Subject Krill). Finally, the geometrically inappropriate comers (noted "N" and $" F$, ,) received, during this control session, an average of $5 \%$ of the first visits (0\% for Subject Crevet and $10 \%$ for Subject Krill). These results suggest that no cue (inside or outside the experimental apparatus) other than the four different colored cues were used by the subjects to reorient.

Table 2 (Experiment 8) provides the results for each subject during the first 10 trials. The individual scores observed for these 10 first trials are not different from the scores obtained

Table 9

Number of Trials (Out of 50) During Experiment 8 for the Subjects as a Function of Their Search Location (Correct, Rotational, Geometrically Inappropriate Corners)

Box

\begin{tabular}{lcrrr}
\multicolumn{1}{c}{ Monkey } & $\mathrm{C}$ & $\mathrm{R}$ & $N$ & $\mathrm{~F}$ \\
\hline Krill & 38 & 7 & 5 & 0 \\
Crever & 34 & 10 & 4 & 2 \\
$\quad$ Average (\%) & 76 & 18 & 3 & 3
\end{tabular}

Note. $\mathrm{C}=$ correct $; \mathrm{R}=$ rotational $\quad N=$ near misses $; \mathrm{F}=$ far misses. 
during the 50 trials of Experiment 6, Krill, $X 2(1, N=60)=0.05$; Crevet, $X 2(l, N=60)=0.54 ; \mathrm{p}>.05$. These results indicate that no improvement of the monkeys' performance took place during Experiment 8.

\section{Discussion}

The subjects were able to correctly locate the rewarded box without ambiguity. By using the four different comer cues, the monkeys were able to distinguish the correct and the geometrically equivalent corer. The control condition shows that no other cue than the corer cues was used by the subjects to reorient inside the experimental apparatus. Thus, we can conclude that when the cues are salient enough, whatever their position, they can be used by the monkeys and help them to correctly reorient in the rectangular room.

\section{General Discussion}

In the present experiments, we investigated the ability of young rhesus monkeys to reorient, after they had been disoriented, in a perfectly symmetrical rectangular environment, by using the geometric and nongeometric features provided by the apparatus. In the first series of experiments, we tested the capacity of 3 monkeys to reorient when only the geometric features of the experimental rectangular room were available (Experiment 1). In Experiments 2 and 3 we tested the orientation of the monkeys by using a large nongeometric feature (either associated or dissociated from the reward box). Our results clearly show that the monkeys can rely on both geometric and nongeometric features to locate the target. In the second series of experiments, we investigated the orientation abilities of the monkeys to use small, distal, and proximal cues either indirectly (Experiment 4) or directly (Experiment 5) associated to the corers of the rectangular room. In both conditions, the monkeys were unable to correctly locate the target. In effect, they still relied only on the geometry of the apparatus. In the third series of these experiments, we investigated the effect of the landmark size on monkeys' orientation abilities. In Experiment 6, the use of a small central cue was not salient enough to allow for a nonambiguous orientation. In Experiments 7 and 8, a larger central or four different corer cues provided sufficient information for correctly orienting the monkeys and allowing them to find the rewarded box without ambiguity.

Our results extend the earlier findings on rats (Cheng, 1986; Margules \& Gallistel, 1988), and nonhuman (Tinklepaugh, 1932) and human primates (Hermer \& Spelke, 1994, 1996; Wang et al., 1999), suggesting that these species are able to reorient on the basis of the geometric features of the environment after disorientation. By testing nonhuman primates in a task similar to that used by Cheng (1986) and Hermer and Spelke (1996), we provide further evidence to the existence of a common orientation mechanism, which is based on the geometry of the environment, found in other mammal species, and more specifically in a species phylogenetically closer to humans than rats. As stated by Cheng (1986), it seems that there is a unit in the mammalian brain that specifically encodes the geometric properties of the environment, such as the metric relations between different spatial elements. This "modular organization" of spatial representations can be understood in the Fodorian meaning of a "module," that is, a rigid structure by which information is required to transit and is automatically processed (Fodor, 1983). As an example, in each of our experiments, the subjects were able to reorient correctly with respect to the geometric relationships that were defining the correct comer (and also its rotational equivalent). Even when monkeys were unable to use nongeometric information (Experiments 4, 5 , and 6), they still relied, at least, on the geometry of the apparatus to find the geometrically correct comers.

Gallistel (1990) suggested that disoriented animals determine their position and heading within a mapped environment by computing the principal axes (or other shape parameters) of the currently perceived environment and compared this information with their spatial representation. By comparing shape parameters, the animal brain determines the spatial information required for making its position and orientation with respect to the currently perceived world, congruent with a position and orientation on its internal spatial representation. If animals compute the heading in this way, then the process of determining heading is effectively a module according to Fodor's (1983) definition. Such a module must be unaffected by sensory data that define properties of the environment other than its macroscopic shape, no matter how relevant those properties may be to the task. The hypothesized computational process is impenetrable to perceptual properties other than shape because shape parameters, such as principal axes, are defined only by the shape of the environment.

Furthermore, the experiments by Hermer and Spelke with young children $(1994,1996)$ suggest that, in the same way, children's reorientation system is unaffected by all but geometric information, even when nongeometric information is available. According to Hermer and Spelke, the limits of this process are overcome during human development. More specifically, Hermer and Spelke assumed that the use of both geometric and nongeometric information provided by the environment after disorientation could be a uniquely human capacity for problem solving. More striking is their hypothesis concerning the use of language for solving such spatial problems. In several articles, these authors (Hermer \& Spelke, 1996; Spelke \& Hermer, 1996; Hermer-Vasquez et al., 1999) proposed that the geometric reorientation process found in rats and children is preserved in adults and is impervious to interference from concurrent language production, whereas the ability to use nongeometric information is vulnerable to such interference. Indeed, they found that performing a verbal shadowing task during disorientation prevented the participants from taking into account nongeometric information for orienting (Spelke \& Hermer, 1996).

Thus, language and more specifically spatial language that children of 2-3 years begin to produce and use (MacWhinney, 1991) appears to provide an especially useful medium for representing conjunctions of spatial and nonspatial properties of the environment. For example, Hermer-Vasquez, Moffet, and Munkholm (2001) showed a correlation between the ability of children to produce and use phrases involving "left" and "right" when verbally describing the hidden location (e.g., "the toy is in the comer to the left of the blue wall") and the ability to correctly reorient in the blue-wall condition. Whereas systems for representing the environmental layout may be confined to capturing geometric information, and systems for representing movable objects may be confined to capturing local properties of objects or surfaces, language can bring these sources of information together, specifying 
that an object or a surface with one set of properties stands in a particular geometric relationship to another object or surface. In sum, language acquired by children (starting at 2-3 years of age) would allow them to perform as well as adults (at 5-7 years of age) in a reorientation task, and no longer as rats.

Showing for the fast time that a mammal species, the rhesus monkey, phylogenetically close to humans, is able to combine both geometric and nongeometric information to reorient (Experiments 2 and 3 ) leads us to cast doubt (a) on the "language hypothesis" in the particular experimental setup we used, and (b) on the proposal that using local features of the environment is a specifically human ability. As a matter of fact, the joint use of both geometric and landmark-based cues by rhesus monkeys suggests that one or several types of spatial processing could either emerge or have evolved and become more flexible with evolution. Such flexibility could have evolved independently of specifically human cognitive features such as symbolic representation, formal reasoning, and, of course, language.

One reason that could explain this evolution is that (a) the foraging behavior in the wild of a monkey is certainly more complex and more difficult than the foraging behavior of a rat, and (b) increasing foraging demands led to elaborate cognitive capacities (see Milton, 1981; Vauclair, 1996). It is clear that, for any mammal, the use of geometric information to reorient in a natural environment is safer because the macroscopic shape of the landscape does not change across seasons. In contrast, there are important changes in the nongeometric properties of the wild, for example, the appearance of trees with or without leaves or snowfall and melting snow (Hermer \& Spelke, 1996). However, for more complex mammals such as nonhuman primates, the ability to use also nongeometric information, such as the color of a mature fruit, could certainly improve their foraging strategies and consequently improve their survival chances.

Alternative explanations of the monkeys' performance in our experimental situation can be advanced. Because each monkey had a single rewarded box during each experiment, another possible explanation of the results observed in Experiments 2 and 3 could be that our subjects developed some specific strategies during the 50 trials of each experiment to succeed in the task. Nevertheless, close examination of the first data shows no improvement in performance across trials (see Table 2 for detailed results), indicating that monkeys were relying on the geometric and nongeometric properties of the environment from the first trials. Furthermore, preliminary results from a study conducted with another primate species, the cotton top tamarin (Miller, Gouteux, Delpolyi, Santos, \& Hauser, 2001) indicate that, in a similar task, these monkeys succeeded within four trials in locating the target by using the nongeometric properties of the environment. Though there are differences between rhesus monkeys and cotton top tamarins, we can reasonably conclude that animals from both primate species are able to use geometric and nongeometric features of the experimental environment in the very first trials. Nevertheless, it could be that subjects used only nongeometric information to find the correct comer when nongeometric information was sufficient to distinguish the correct corner from its geometrically opposite. In such a case, subjects could then use self-referent spatial information, namely by distinguishing right and left side when facing the landmark. However, according to our results and to the modularity hypothesis, it would be surprising that geometric information was not used, even when the environment provided nongeometric cues. In effect, the use of geometry appears to be a predominant element for spatial reorientation. Thus, in each experiment we ran, monkeys used geometry to reorient even when cues were available (but not used) in the experimental apparatus. This finding led us to claim that when monkeys use nongeometric spatial information, they certainly also use geometric information.

When more precise local information was provided (a small angular cue in Experiments 4 and 5), the monkeys were not able to correctly reorient by using this unambiguous spatial information. In these cases, they were acting as if no spatial information other than the shape of the experimental environment was present: Monkeys were searching with equal frequencies in the correct and geometrically correct locations. These results lead us to suggest several alternative interpretations. First of all, the cues might not have been noticed by the subjects (that would explain why they did not use them to reorient). However, the analysis of videotapes shows that in many trials the monkeys displayed investigatory reactions to these comer cues. For example, they often took a look at or touched them during trials. Thus, it seems unlikely that monkeys had not paid attention to these cues.

Another explanation of the errors made during Experiments 4 and 5 could be due to a lack of motivation of the subjects. However, rhesus monkeys were sufficiently motivated (and attended) to search correctly in the geometrically appropriate comer, indicating that they correctly encoded and used the geometry of the room to reorient.

A more plausible explanation would be that these angular cues were not considered by the monkeys as stable or nonmovable. According to Biegler and Morris (1993, 1996), the relative stability of landmarks is an important factor in the development of spatial behaviors. It is generally thought that an object must be considered as immovable in order to be used as a landmark in navigation tasks (for an example with young children, see Acredolo, 1990). In Experiment 4, the two geometric comer cues were shown before disorientation but were removed afterward. This experimental procedure may have led the monkeys to consider the angular cues as movable and thus not reliable for reorientation. This kind of explanation is also suggested by Learmonth, Newcombe, and Huttenlocher (1998) to explain Hermer and Spelke's results with children. According to Learmonth et al. (1998), the nongeometric cues in Hermer and Spelke's experiments appear to be nonpermanent because children could see the experimenter attaching the blue fabric to one of the white walls, but this fabric was sometimes there and sometimes not there (because the manipulation was performed within subjects). In another study by Hermer and Spelke (1996), children were allowed to play with a toy truck and a toy teddy bear, that is, with movable objects that were used as landmarks by the experimenter. On the basis of these data, Learmonth et al. have replicated Hermer and Spelke's experiments with the hypothesis that children of the same age would reorient by using more permanent appearing landmarks, such as a door or a bookcase. Their data show that children between 18 and 24 months can, to some extent, use the door and the bookcase to reorient because they found the hidden toy reliably more often than chance and search reliably more often at the correct comer than at the rotationally equivalent comer.

Concerning the results of Experiment 5, the only explanation that accounts for the lack of utilization of local cues for reorienting 
is their small size. As a matter of fact, when we tested the effects on performance of cue size (Experiments 6 and 7), we observed that monkeys were able to correctly reorient only with large or intermediate-sized cues (entire wall of Experiments 2 and 3; Experiment 7) but not with the small-size cues used in Experiment 6 (and also in Experiments 3 and 4 but at different locations). Data of Experiments 5 and 8 support this view. Indeed, monkeys were confronted to the same experimental environment, except for the size of the corner cues. In Experiment 4 (small comer cues), subjects failed to correctly locate the rewarded box, but with larger cues, they perfectly succeeded in the task.

Taken together, these results strongly suggest that the use of nongeométric information to reorient is tightly related to physical intrinsic characteristics (e.g., the size) and to the stable status of the informative elements. According to our data (Experiments 6, 7, and 8), even when the presence of a cue is obvious for the monkeys, they are unable to use it to reorient unless its size is large enough. It should be mentioned that children also seem to use nongeometric cues only when these cues appear as stable landmarks and are also of a sufficiently large size. (Even if the truck toy used in Hermer \& Spelke's experiment was not considered as immobile, we can also suggest that its size could have played an important role for being treated as a landmark.)

In short, the data of the present experiments bring some questions to the notion of a geometric module for spatial processing. Some studies have shown that a conspicuous local cue (a colored wall, for instance) is neglected by toddlers or by human adults who perform a dual-task (Hermer-Vasquez et al., 1999). Those data fit the properties of such a module well. However, in other experiments salient physical features of the local cues appear likely to induce a further processing (Acredolo, 1990). In the latter case, an explanation in terms of modular processing is inadequate, because modules are defined with properties such as encapsulation or impenetrability. We propose that the salience of local information may lead subjects to take it into account, but only in a second phase, that is, after a modular but sometimes insufficient processing. The fact that geometric features are never neglected suggests that their processing is automatic and systematic. In contrast, using local information to disambiguate the situation would depend on the properties of the local cues themselves and perhaps also on other interacting factors such as motivation, age of the subjects, and so forth.

Also, concerning our experimental situation, an alternative explanation to the modularity hypothesis could be formulated. We suggest that instead of automatically processing the geometric information of the environment, the rhesus monkeys could use a more basic procedure to reorient. This procedure may be based on some perceptual mechanisms that could encode different types of spatial information, according to their respective salience in the environment. In effect, when no landmarks were available in the experimental room (e.g., Experiment 1), monkeys relied on the most salient and perceptible spatial information available, namely the shape of the room. This geometric information was also the only information to be used even when small-size cues were available in the room (Experiments 4, 5, and 6), suggesting that when the salience or weight of perceptible information is not sufficient, monkeys ignore it. This prediction is confirmed by results of Experiments 2, 3, 7, and 8. In these experiments, the relative weight of nongeometric information compared with the weight of geometric information provided by the shape of the room is sufficient to allow the monkeys to rely simultaneously on these two types of spatial information, leading the reorientation mechanisms to be more efficient. In sum, we suggest that, in addition to referring to a modular processing, our results could also be explained in terms of perceptual attractiveness.

Despite the fact that nonhuman primates are able to combine geometric and nongeometric spatial information without language, this finding does not allow us to conclude that humans do not solve the disorientation problem by encoding the available information linguistically. In fact, data from human adults and children (see Hermer-Vasquez et al., 1999) suggest that language is necessary to human beings for combining these two different aspects of core knowledge. This specific human mechanism, which is based on language, may have emerged across evolution and could account for the better flexibility of the human being with respect to other mammals. However, this hypothesized mechanism and its functioning required further investigations. It is precisely the comparative approach with nonhuman primates that could help us to better understand this human peculiarity.

\section{References}

Acredolo, L. P. (1990). Behavioral approaches to spatial orientation in infancy. In A. Diamond (Ed.), Annals of the New York Academy of Sciences: Vol. 608. The development and neural bases of higher cognitive function (pp. 596-612). New York: New York Academy of Sciences.

Benhamou, S., \& Poucet, B. (1998). Landmark use by navigating rats (Rattus norvegicus): Contrasting geometric and featural information. Journal of Comparative Psychology, 112, 317-322.

Biegler, R., \& Moms, R. G. M. (1993, February 18). Landmark stability is a prerequisite for spatial but not discrimination learning. Nature, 361, 631-633.

Biegler, R., \& Moms, R. G. M. (1996). Landmark stability: Studies exploring whether the perceived stability of the environment influences spatial representation. Journal of Experimental Biology, 199, 187-193.

Cheng, K. (1986). A purely geometric module in the rat's spatial representation. Cognition, 23, 149-178.

Cheng, K. (1987). Rats use the geometry of surfaces for navigation. In P. Ellen \& C. Thinus-Blanc (Eds.), Cognitive processes and spatial orientation in animals and man (pp. 153-159). Dordrecht, the Netherlands: Martinis Nijhoff.

Cheng, K., \& Gallistel, C. R. (1984). Testing the geometric power of an animal's spatial representation. In H. L. Roitblat, T. G. Bever, \& H. S. Terrace (Eds.), Animal cognition (pp. 409-423). Hillsdale, NJ: Erlbaum. Dudchenko, P. A., Goodridge, J. P., Seiterle, D. A., \& Taube, J. S. (1997). Effects of repeated disorientation on acquisition of spatial tasks in rats: Dissociation between the appetitive radial arm maze and the aversive water maze. Journal of Experimental Psychology: Animal Behavior Processes, 23, 194-210.

Fodor, J. (1983). The modularity of mind. Cambridge, MA: MIT Press. Gallistel, C. R. (1990). The organization of learning. Cambridge, MA: MIT Press.

Gouteux, S., Vauclair, J., \& Thinus-Blanc, C. (1999). Reaction to spatial novelty and exploratory strategies in baboons. Animal Learning and Behavior, 27, 323-332.

Greene, C. M., \& Cook, R. G. (1997). Landmark geometry and identity controls spatial navigation in rats. Animal Learning \& Behavior, 25, 312-323.

Hermer, L. (1997). Cognitive flexibility as it emerges over development and evolution: The case of two navigational tasks in humans. (Unpublished doctoral dissertation, Cornell University, 1997). 
Hermer, L., \& Spelke, E. (1994, July 7). A geometric process for spatial reorientation in young children. Nature, 370, 57-59.

Hermer, L., \& Spelke, E. (1996). Modularity and development: The case of spatial reorientation. Cognition, 61, 195-232.

Hermer-Vasquez, L., Moffet, A., \& Munkholm, P. (2001). Language, space, and the development of cognitive flexibility in humans: The case of two spatial memory tasks. Manuscript in preparation.

Hermer-Vasquez, L., Spelke, E., \& Katsnelson, A. (1999). Source of flexibility in human cognition: Dual-task studies of space and language. Cognitive Psychology, 39, 1, 3-36.

Joubert, A., \& Vauclair, J. (1986). Reaction to novel objects in a troop of Guinea baboons: Approach and manipulation. Behaviour, 96, 92-104.

Kamil, A. C., \& Jones, J. E. (1997). The seed-storing corvid Clark's nutcracker learns geometric relationships among landmarks. Nature, 390,276-279.

Kelly, D. M., Spetch, M. L., \& Heth, C. D. (1998). Pigeon's encoding of geometric and featural properties of a spatial environment. Journal of Comparative Psychology, 112, 259-269.

Knierim, J. J., Kudrimoti, H. S., \& MacNaughton, B. L. (1995). Hippocampal place fields, the internal compass, and the learning of landmark stability. Journal of Neuroscience, 15, 1648-1659.

Learmonth, A., Newcombe, N., \& Huttenlocher, J. (1998). Disoriented children use landmarks as well as geometry to reorient. Paper presented at the meeting of the Psychonomics Society, Dallas, TX

Lopez, J. C., Broglio, C., Rodriguez, F., Thinus-Blanc, C., \& Salas, C. (1999). Memory strategies in goldfish trained in a spatial constancy task versus a cued one. Animal Cognition, 2, 109-120.

MacNaughton, B. L., Knierim, J. J., \& Wilson, M. A. (1994). Vector encoding and the vestibular foundations of spatial cognition: Neurophysiological and computational mechanisms. In M. Gazzaniga (Ed.), The cognitive neurosciences (pp. 587-595). Cambridge, MA: MIT Press.

MacWhinney, B. (1991). The CHILDES Project: Tools for analyzing talk. Hillsdale, NJ: Erlbaum.

Margules, J., \& Gallistel, C. R. (1988). Heading in the rat: Determination by environmental shape. Animal Learning and Behavior, 16, 404-410.

Menzel, E. W. (1973). Chimpanzee spatial memory organization. Science, 182,943-945.

Menzel, E. W. (1978). Cognitive mapping in chimpanzees. In S. H. Hulse,
H. Fowler, \& W. K. Honig (Eds.), Cognitive processes in animal behavior (pp. 375-422). Hillsdale, NJ: Erlbaum.

Miller, C. T., Gouteux, S., Delpolyi, A., Santos, L. R., \& Hauser, M. D. (2001). Primate spatial representation: Coordinated use of geometric and landmark information by cotton top tamarins. Manuscript in preparation.

Milton, K. (1981). Distribution pattern of tropical plant foods as an evolutionary stimulus to primate mental development. American Anthropologist, 83, 534-548.

Spelke, E. S., \& Hermer, L. (1996). Cognition and language. In R. Gelman \& T. Kit-Fong Au (Eds.), Perceptual and cognitive development (pp. 71-114). New York: Academic Press.

Suzuki, S., Augerinos, G., \& Black, A. H. (1980). Stimulus control of spatial behavior on the eight-arm maze in rats. Learning and Motivation, 217,150-154.

Thinus-Blanc, C. (1996). A nimal spatial cognition: Behavioral and neural approches. Singapore: World Scientific Publications.

Thinus-Blanc, C., Bouzouba, L., Chaix, K., Chapuis, N., Durup, M., \& Poucet, B. (1987). A study of the spatial parameters encoded during exploration in hamsters. Journal of Experimental Psychology: Animal Behavioral Processes, 13, 418-427.

Tinklepaugh, O. (1932). Multiple delayed reaction with chimpanzees and monkeys. Journal of Comparative Psychology, 13, 207-243.

Tolman, E. C. (1948). Cognitive maps in rats and men. Psychological Bulletin, 55, 189-208.

Vallortigara, G., 7anforlin, M., \& Pasti, G. (1990). Geometric modules in animals' spatial representations: A test with chicks (Gallus gallus domesticus). Journal of Comparative Psychology, 104, 248-254.

Vauclair, J. (1996). Animal cognition: Recent developments in modern comparative psychology. Cambridge, MA: Harvard University Press.

Wang, R. F., Hermer, L., \& Spelke, E. S. (1999). Mechanisms of reorientation and object localization by children: A comparison with rats. Behavioral Neuroscience, 113, 475-485.

Received June 30, 1999

Revision received July 11, 2000 Accepted September 25, $2000 \mathrm{X}$ 